\title{
Application of molecular weight regulators for the synthesis of sodium polyacrylate thinners of mineral suspensions.
}

\author{
Belov D.A.,** \\ ${ }^{1}$ Belarusian State University, Leningradskaya str. 14, Minsk, Belarus. \\ *Correspondence: beldis@tut.by
}

\begin{abstract}
The synthesis of additives for thinning mineral suspensions based on sodium polyacrylate was carried out. The effect of molecular weight regulators on the molecular weight characteristics of the polymer and the effect of such polymers on the rheological properties of suspensions was studied. Sodium acrylate polymers are synthesized by free radical polymerization in aqueous solution using molecular weight regulators. The molecular weight characteristics of the polymeric samples were estimated by viscometry using Mark-Houwink-Kuhn-Sakurada (MHKS) equation. Synthesized polymers were used as thinners ceramic slurries, prepared according to the recipe of the enterprises producing ceramic products. The thinning ability of polymer samples with different molecular weights was estimated using an Engler viscometer from the time of the ceramic slurry flow. The influence of the type and amount molecular weight regulator on polyacryates was revealed. It was found that molecular weight synthesized samples was in the range of 21000 - 91000. It was determined that samples with a molecular weight of 28000 - 35000 synthesized using mercaptoethanol (at a dosage of $0.5-1.5 \%$ by weight of the monomer) provide optimal fluidity to the ceramic slurry.
\end{abstract}

Key words: mineral suspension, thinner, free radical polymerization, molecular weight regulator, sodium polyacrylate. 


\section{Introduction}

Slurries based on clay minerals are used for the production of sanitary ware and ceramic tiles. The technology for the production of such items is very sensitive to the physical characteristics of the ceramic suspension. First of all, the requirement is high fluidity, with a limited amount of water content in this mineral suspension. The increased water content makes the slurry more fluid, but leads to an increase in energy consumption for moisture removal from finished products. Also, a high water content in the slurry leads to a deterioration in its properties [1, 2]. In this regard, there is a problem of synthesis chemical additives for thinning mineral suspensions. Such additives can be: salts of polyphosphoric, silicic and humic acids, etc. Nevertheless, synthesis of new additives for thinning slurries remains relevant $[1,5,6]$. One of the promising directions is the use of polymers and, in particular, sodium polyacrylate in this role. The ability of water-soluble acrylic polymers to prevent the aggregation of the mineral part of the suspension deflocculation, leads to a decrease of the viscosity of the mineral suspension, which makes it possible to reduce the energy consumption for its preparation, or to achieve the required viscosity without increasing the water content [3-6]. Thinning ability of acrylic polymers in mineral slurries depends on the monomer composition and molecular weight distribution. To regulate the chain length in radical polymerization, molecular weight regulators are used. The most effective are sulfur containing compounds that regulate $\mathrm{MM}$ also by the chain transfer mechanism [8-14].

Aim of the work: synthesis of sodium polyacrylate samples with different molecular weights using mercaptoethanol, thioacetic acid and sodium sulfite as molecular weight regulators; comparative analysis of the effectiveness of molecular weight regulators; determination of the thinning ability of sodium polyacrylate in mineral suspensions; establishing the optimum ratio of components to obtain an effective thinning additive. 


\section{Materials and Methods}

For the synthesis of polymer compounds, we used: distilled water, acrylic acid (AA) (Sigma-Aldrich), ammonium persulfate (PSA) (Aldrich), sodium carbonate (Sigma-Aldrich), sodium sulfite (SS) (Sigma-Aldrich), mercaptoethanol (ME) (Sigma-Aldrich), thioacetic acid (TA) (Sigma-Aldrich). The reagents were used without preliminary purification.

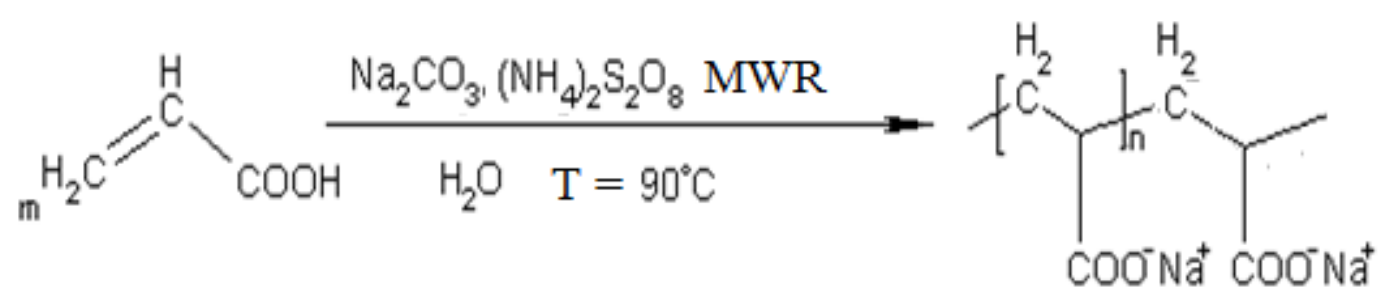

Figure. 1 - Sodium Polyacrylate Synthesis

In a sealed reaction flask with distilled water heated to $90^{\circ} \mathrm{C}$, solutions of acrylic acid, ammonium persulfate (PSA) and a regulator (MWR) (if necessary) were simultaneously introduced, the rate of introduction of reagents was selected so as to prevent boiling of the reaction medium due to the exothermic effects of polymerization. The synthesis was carried out for 4 hours, then cooled and neutralized with sodium carbonate. The amount of water used in the synthesis was calculated to obtain a $20 \%$ final sodium polyacrylate solution. Four series of polymer samples based on sodium acrylate were synthesized: without a molecular weight regulator, using mercaptoethanol, sodium sulfite, and thioacetic acid as MM regulators.

Intrinsic viscosity was determined according to the method described in [7], for this, a $0.1 \%$ polymer solution in $3 \% \mathrm{NaCl}$ was prepared. The measurements were carried out at $25^{\circ} \mathrm{C}$ in a capillary viscometer with a hanging level (capillary diameter $0.56 \mathrm{~mm}$ ), constant in Mark-Houwink-Kuhn-Sakurada (MHKS) equation $\alpha=0.6200 ; \mathrm{K}=0.0005270$. 
For the slurry, the following components were used: sand, clay (NizhneUvelskaya semi-acid refractory kaolinite), water, synthesized sodium polyacrylate (as a thinner). The ratio of the components equal recipe used in the enterprises producing ceramic products. The slurry is loaded into a ball mill for 30 minutes, after preparation slurry fluidity is determined on an Engler viscometer, which is a metal cylinder with a spherical bottom with a hole. Slurry fluidity - the time during which this mineral suspension flows out of the viscometer. When determining the fluidity with one slurry, 3 experiments are carried out and the average value is calculated. The optimum fluidity of the clay slurry used in the production of ceramics is $17-19 \pm 1$ seconds.

\section{Results}

The molecular mass of compounds obtained by the method of radical polymerization depends on many factors: the nature of the monomer, initiator, solvent, temperature, also on the ratio of components, etc. To obtain polymers with a certain MW value, the concentration of the polymerization initiator can be varied (the higher the initiator concentration, the higher the polymerization rate, but the lower the molecular weight of the resulting polymer). However, this method increases the economic costs of production, and also promotes branching and crosslinking reactions of the polymer chain, as a consequence of this, the molecular weight distribution of the polymer is very wide.As can be seen from table 1 , even with a very significant content of the initiator $(1.2 \mathrm{~g}$ PSA to $14.2 \mathrm{~g} /$ AA) more than $8 \%$ by weight of the monomer, intrinsic viscosity and molecular weight is quite high. 
Table 1. - Mass ratio of components in the synthesis of sodium polyacrylate.

\begin{tabular}{|c|c|c|c|}
\hline Sample & $\mathbf{m}(\mathbf{A A}), \mathbf{g}$ & $\mathbf{m}(\mathbf{P S A}), \mathbf{g}$ & $\mathbf{M M}$ \\
\hline 1 & 14,20 & 1,20 & 45000 \\
\hline 2 & 14,20 & 0,80 & 49000 \\
\hline 3 & 14,60 & 0,40 & 60000 \\
\hline 4 & 14,80 & 0,20 & 68000 \\
\hline 5 & 14,86 & 0,14 & 80000 \\
\hline 6 & 14,89 & 0,11 & 91000 \\
\hline
\end{tabular}

To control the molecular weight, it is possible to introduce into the reaction mixture substances that decrease the molecular weight of the polymer at a constant concentration of the initiator.

To establish the effect of the regulator content, the amount of initiator $0.40 \mathrm{~g}$ PSA in $14.6 \mathrm{~g}$. AA (2.7\% by weight of the monomer) was fixed, and different amounts of molecular weight regulator were introduced. The ratios of the components of synthesis for three series of samples are presented, in table. 2 - 4.

Table 2. - Mass ratio of components in the synthesis of sodium polyacrylate using sodium sulfite (SS) as a molecular weight regulator.

\begin{tabular}{|c|c|c|c|}
\hline Sample & $\mathbf{m}(\mathbf{A K}), \mathbf{g}$ & $\mathbf{m}(\mathbf{S S}), \mathbf{g}$ & $\mathbf{M M}$ \\
\hline 7 & 14,60 & 0,04 & 65000 \\
\hline 8 & 14,60 & 0,12 & 61000 \\
\hline 9 & 14,60 & 0,28 & 58000 \\
\hline 10 & 14,60 & 0,32 & 59000 \\
\hline 11 & 14,60 & 0,40 & 51000 \\
\hline 12 & 14,60 & 0,50 & 46000 \\
\hline 13 & 14,60 & 0,60 & 39000 \\
\hline 14 & 14,60 & 0,80 & 32000 \\
\hline
\end{tabular}


Table 3. - Mass ratio of components in the synthesis of sodium polyacrylate using mercaptoethanol (ME) as a molecular weight regulator.

\begin{tabular}{|c|c|c|c|}
\hline Sample & $\mathbf{m}(\mathbf{A K}), \mathbf{g}$ & $\mathbf{m}(\mathbf{M E}), \mathbf{g}$ & $\mathbf{M M}$ \\
\hline 15 & 14,60 & 0,04 & 40000 \\
\hline 16 & 14,60 & 0,08 & 35000 \\
\hline 17 & 14,60 & 0,2 & 28000 \\
\hline 18 & 14,60 & 0,28 & 27000 \\
\hline 19 & 14,60 & 0,40 & 23000 \\
\hline
\end{tabular}

Table 4 - Mass ratio of components in the synthesis of sodium polyacrylate using thioacetic acid(TA) as a molecular weight regulator.

\begin{tabular}{|c|c|c|c|}
\hline Sample & $\mathbf{m}(\mathbf{A K}), \mathbf{g}$ & $\mathbf{m}(\mathbf{T A}), \mathbf{g}$ & MM \\
\hline 20 & 14,60 & 0,04 & 38000 \\
\hline 21 & 14,60 & 0,08 & 36000 \\
\hline 22 & 14,60 & 0,2 & 31000 \\
\hline 23 & 14,60 & 0,28 & 22000 \\
\hline 24 & 14,60 & 0,40 & 21000 \\
\hline
\end{tabular}

In all cases, with the addition of a regulator, the MM decreases. Despite the difference in the structure of substances, all three regulators can be used as components of the reaction mixture to control the molecular mass of the resulting polymer. The mechanism of action of these substances, is well studied [6-8], but the comparative efficiency of these compounds and the effect of their effect on the properties of the target product, which is used to thinning clay suspensions, is of scientific interest [11-14].

An increase in the concentration of sodium sulfite and mercaptoethanol and thioacetic acid leads to a fall of values intrinsic viscosity and MM. In the case of sodium sulfite, the fall of MM values is less. So, this compound shows the least efficiency as an MM regulator. 
Characteristics of the slurry are presented in Table 5. It was shown the dependence slurry fluidity on the molecular weight of sodium polyacrylate samples, synthesized with different molecular weight regulators.

Table 5 - The dependence of fluidity ceramic slurry on the MM and MWR of synthesized polymers

\begin{tabular}{|c|c|c|c|}
\hline Sample & MWR & MM & Slurry fluidity, sec \\
\hline 1 & \multirow[t]{6}{*}{-} & 45000 & $-*$ \\
\hline 2 & & 49000 & $-*$ \\
\hline 3 & & 60000 & -* \\
\hline 4 & & 68000 & $-*$ \\
\hline 5 & & 80000 & $-*$ \\
\hline 6 & & 91000 & $-*$ \\
\hline 7 & \multirow[t]{8}{*}{ SS } & 65000 & $-*$ \\
\hline 8 & & 61000 & $-*$ \\
\hline 9 & & 58000 & $-*$ \\
\hline 10 & & 59000 & $-*$ \\
\hline 11 & & 51000 & $-*$ \\
\hline 12 & & 46000 & 26.5 \\
\hline 13 & & 39000 & 19.5 \\
\hline 14 & & 32000 & $-*$ \\
\hline 15 & \multirow[t]{5}{*}{$\mathrm{ME}$} & 40000 & $-*$ \\
\hline 16 & & 35000 & 19 \\
\hline 17 & & 28000 & 18 \\
\hline 18 & & 27000 & $-*$ \\
\hline 19 & & 23000 & $-*$ \\
\hline 20 & \multirow[t]{5}{*}{ TA } & 38000 & $-*$ \\
\hline 21 & & 36000 & 20 \\
\hline 22 & & 31000 & 20.5 \\
\hline 23 & & 22000 & $-*$ \\
\hline 24 & & 21000 & $-*$ \\
\hline
\end{tabular}

*Slurry does not flow out of the viscometer. 
As shown in Table 5, when sodium polyacrylate synthesized without regulator $\mathrm{MM}$ or with sodium sulfite, the resulting samples do not have thinning properties. For the samples synthesized using mercaptoethanol and thioacetic acid, an extreme dependence of the thinning efficiency on the molecular weight was observed. The best result is achieved by MM $28000-35000$ when $0.08-0.2 \mathrm{~g}$ of mercaptoethanol adding to $14.6 \mathrm{~g}$ of monomer, which corresponds to $0.5-1.5 \%$.

The processes leading to a change in the rheological properties of the slurry occur in the electric double layer (DES). DES is formed on the surface of clay particles as a result of the adsorption of ions from the solution, the dissociation of the surface compound, or the orientation of polar molecules at the phase boundary. According to modern concepts, the electric double layer can be considered as a spatial separation of charges of opposite sign in the interphase layer. Ions directly bound to the surface are potential-determining. The charge of this layer is compensated by the charge of the ions of the second layer - counterions [6]. The thinning effect exhibited by the sodium polyacrylate images in the clay suspension can be explained by the following reasons:

1. Cation exchange. To increase the electric double layer and, consequently, to increase the repulsive effect, it is necessary to replace divalent cations with monovalent ones. The negative surface charge of clay minerals cannot be completely screened by divalent cations. The exchange of divalent cations for monovalent ones, for example, sodium, leads to better overlapping of negative charges and to an increase in the double electric layer. Agglomeration of particles is prevented, viscosity is reduced

2. Steric repulsion. Dispersant with functional groups act on the mechanism of steric repulsion. These functional groups act as spacers between the mineral particles. The adsorption of a dispersant on the surface of raw material particles causes repulsion between them, which leads to a uniform distribution of fine particles in a dispersion medium, in which all particles are ideally located at an equal distance from the nearest neighbors. 
3. Complexation. This mechanism consists in the addition of agents that bind free divalent cations before they can contact the surface of the starting material particle and cause flocculation.

The number of carboxyl groups, determining the first and third mechanisms of deflocculating action, did not change upon the addition of equal amounts of polyacryates with different molecular weights in slurry. So it can be concluded that, at a certain molecular weight corresponding to MM 28000 - 35000, the conformation and size of sodium polyacrylate chain tangles provide maximum steric repulsion. This was achieved using mercaptoethanol and, with less potency, with thioacetic acid as a regulator.

\section{Conclusions}

Polymers and copolymers of sodium acrylate were obtained at various ratios of components, and the molecular weight of all samples was determined. MW values were obtained in the range of 21000 - 65000 using molecular weight regulators and 45000-91000 without them.

Analysis of the effectiveness of molecular weight regulators was carried out and it was found that organic substances mercaptoethanol and thioacetic acid is the more effective.

The thinning ability of sodium acrylate polymers in mineral suspensions for production of ceramic products has been studied. It was found that in the case of sodium acrylate polymers, mercaptoethanol can be used as a regulator, at a dosage of $0.5-1.5 \%$ of the monomer weight. The macromolecules of polyacrylate in this case have MM 28000 - 35000, the optimal size and conformation of chains, which provide optimal fluidity of the mineral suspension.

\section{References}


1. Modern Ceramic Engineering: Properties, Processing, and Use in Design, Fourth Edition. D.W. Richerson, W. E. Lee. CRC Press. 2018, P.812.

2. R. W. Rice. Ceramic Fabrication Technology. CRC Press. 2002, P 376

3. M. Hashiba, O. Sakurada, M. Itho, T. Takagi, K. Hiramatsu, Y. Nurishi. Effectiveness of a dispersant for the thickening of alumina slurries whilst retaining the fluidity. Journal of Materials Science. 1993, 28, 4456-4460.

4. Pishch, I. V., Klimosh Yu. A., Gapanovich E. I. Flow properties of slip for producing ceramic sanitary ware. Glass and Ceramics. 2006, Vol. 63, N 7-8. P. 259-261.

5. Madhura Bhalerao, Vijay Y Karadbhajne. Polymeric Surfectants. Its Evolution and Review. International Journal of ChemTech Research. 2019, Vol.12, №.1, P. 183-190.

6. S.Sōmiya. Handbook of advanced ceramics: materials, applications, processing, and properties. Amsterdam. Academic Press. 2013, P. 1240.

7. Rabek Y. Experimental methods in polymer chemistry. Moscow.: Mir, 1983. ch. 2, 480 p.

8. J.Yina, X.Liua, J. Chena, S. Leeb, Z. Huang. Polyacrylic acid, a highly efficient dispersant for aqueous processing of tantalum carbide. Ceramics International. 2017, Vol. 43, N 4, P. 3654-3659

9. Y. Pang, S. Guo, Y. Deng, A. Wang. Synthesis and application performance of a dispersant for ceramic slurry. Polymeric Materials Science and Engineering. 2012, 28(11), P. 1-4

10. Guanghua Huang Zhidong Pan Yanmin Wang. Synthesis of sodium polyacrylate copolymers as water-based dispersants for ultrafine grinding of praseodymium zirconium silicate. Colloids and Surfaces A: Physicochemical and Engineering Aspects. 2018, Vol. 558, P. 591-599

11. J. Loiseau, N. Dolerr, J.M. Suau, J.B. Egraz, M.F. Llauro, C. Ladaviere. Synthesis and Characterization of Poly(acrylic acid) Produced by RAFT Polymerization. Application as a Very Efficient Dispersant of $\mathrm{CaCO}_{3}$, Kaolin, and $\mathrm{TiO}_{2}$. Macromolecules. 2003, 36, 3066-3077. 
12. Sh.N. Zhumagaliyeva, R.S. Iminova, G.Zh. Kairalapova, M.M. Beysebekov, M.K. Beysebekov, Zh.A. Abilov. Composite Polymer-Clay Hydrogels Based on Bentonite Clay and Acrylates: Synthesis, Characterization and Swelling Capacity. Eurasian Chemico-Technological Journal. 2017, 19, 279-288.

13. C. Ladaviere, N. Dolerr, J.P. Claverie. Controlled Radical Polymerization of Acrylic Acid in Protic Media. Macromolecules. 2001, 34, 5370-5372

14. E. A. Egorova, V. P. Zubov, I. V. Bakeeva, E. V. Chernikova, E. A. Litmanovich. Controlled Synthesis of Oligomeric Poly(acrylic acid) and Its Behavior in Aqueous Solutions. Polymer Science, Ser. A, 2013, Vol. 55, No. 9, pp. $519-525$. 